\title{
A Survey of Bath Time Incidents Experienced by Mothers and Families of Children Aged 18 Months
}

\author{
Sachi Chikazawa',2, Ayako Sasaki ${ }^{1}$ \\ ${ }^{1}$ Faculty of Nursing, School of Nursing, Osaka Medical and Pharmaceutical University, Osaka, Japan \\ ${ }^{2}$ Doctoral Program, Graduate School of Nursing, Osaka Medical and Pharmaceutical University, Osaka, Japan \\ Email: sachi.chikazawa@ompu.ac.jp
}

How to cite this paper: Chikazawa, S. and Sasaki, A. (2021) A Survey of Bath Time Incidents Experienced by Mothers and Families of Children Aged 18 Months. Health, 13, 1071-1090.

https://doi.org/10.4236/health.2021.131008 $\underline{0}$

Received: September 5, 2021

Accepted: October 9, 2021

Published: October 12, 2021

Copyright $\odot 2021$ by author(s) and Scientific Research Publishing Inc. This work is licensed under the Creative Commons Attribution International License (CC BY 4.0).

http://creativecommons.org/licenses/by/4.0/

\begin{abstract}
Purpose: The potential dangers of infant bathing have an effect not only on the infant's body and life but also on the formation of emotional bonds in the parent-child relationship. This study will explore actual dangers experienced by mothers and families. Method: We distributed an anonymous, self-reported questionnaire at infants' 18-month health checkup, which was collected via postal service. 304 valid responses were received and formed our sample data. Descriptive statistical analysis was performed for each category surveyed. Inferential statistics were used to compare the dangerous incidents experienced with regard to differences between primipara/multipara and the presence/absence of guidance concerning such incidents. This study was approved by the research ethics committees of the affiliated university. Results: $70 \%$ of mothers and families experienced incidents while bathing their infants, with the most common incidents consisting of, in order of frequency, near immersion of the face, actual immersion of the face, and falling into the bath. The most common situations reported when incidents occurred were bathing the infant by oneself, the infant moving unexpectedly, or taking care of other children, in that order. Approximately $70 \%$ of mothers and families had not received instruction on the potential dangers or how to prevent them. Conclusion: This study has elucidated the dangerous situations experienced in the course of bathing infants in the home and the measures taken to prevent such situations. Instructive tools are needed which can be used to easily provide mothers and families with correct information.
\end{abstract}

\section{Keywords}

Bath Time, Incidents, Experience, Children 


\section{Introduction}

Along with increasing nuclearization of families and a declining birthrate, Japan has seen an increase in men and women who have had little experience dealing with young children becoming parents, as well as an increase in parents who lack the knowledge and skills that pertain to raising children [1] [2]. Furthermore, a weakening of community ties means fewer opportunities for support or the exchange of information [3].

Caring for an infant involves a number of elements, including feeding, putting the infant to sleep, changing diapers, and bathing. Bathing in particular is a multifaceted process involving a number of steps, including changing clothes, washing, keeping hold of the infant, and moisture management. Moreover, bathing space is often limited, and many of the steps are independent actions that demand considerable attention and dexterity. It is also necessary to monitor the infant's condition and adapt bathing methods accordingly. In recent years, the length of post-birth hospital stays at Japanese medical facilities has been shortened [4] to an extent that almost no instruction on proper bathing techniques is given [5]. As a result of this situation, families lack adequate knowledge and practice, making safe and comfortable bathing a difficult undertaking.

As there is an element of danger when bathing, unexpected accidents are known to occur. According to a 2019 demographic survey conducted by the Ministry of Health, Labour, and Welfare, unforeseen accidents were the $3^{\text {rd }}$ leading cause of death among infants under one year of age [6]. Incidents that occurred during bathing included life-threatening accidents such as drowning, falls, and burns. The number of cases of near drownings during bath time was higher for infants under one year of age than for other age groups, and fatal accidents did occur [7]. In the current state of Japanese society, parents can hardly avoid situations in which they must bathe their infant without the help of another or in which they have to take care of multiple children at the same time. Inexperience and impaired judgement due to fatigue are also among the many factors which lead to dangerous accidents. Bathtime incidents can be supposed to include drops due to slippery hands, soup getting in the eyes, drowning in the tub, and burns. According to Heinrich's triangle, 300 minor incidents occur for every major injury. It can be assumed that a large number of families experience close calls in the course of their daily bathing routine that go unreported. As existing reports are made up mostly of more serious accidents, the statistics on such close calls are unclear [7]. In a survey on mothers' worries about bathing their infants, over half reported they had concerns. $36.8 \%$ of primipara and $17.1 \%$ of multipara reported feeling a sense of danger associated with bathing [5]. These results highlighted a need for support from nursing professionals regarding safe bathing practices and the prevention of accidents.

Bathing is a daily practice with many purposes, for not only physical aspects like hygiene, observation of the state of the body, and improvement of metabolism, but also for mental aspects like establishing a life rhythm and parent-child 
intimacy. Bathtime dangers prevent the fulfillment of these goals, potentially threatening the formation of emotional bonds and having negatively influencing the parent-child relationship, in addition to the immediate dangers to the infant's body and life.

From the above points, we have concluded that measures must be taken to assist mothers and their families in safely and confidently bathing their newborns. With this in mind, we conducted this study with the purpose of exploring the dangers related to infant bathing experienced by mothers and families in Japan that are not yet apparent.

\section{Method}

\section{1) Terminology}

a) Infant: A child of less than one year of age.

b) Bathing: The washing of an infant in a bathtub, including related acts such as preparation, dressing, washing, holding the infant's body, and post-bath care.

c) Dangers/Incidents: Bathtime accidents which threaten the life or health of the child, as well as close calls which did not have a lasting effect on the child's life or health.

\section{2) Study design \& survey period}

a) Study Design

Fact-finding survey.

b) Survey Period

June 12, 2020-September 23, 2020.

\section{3) Subjects}

Mothers who brought their infants for their 18-month health checkup were surveyed to investigate the conditions around infant bathing.

\section{4) Method of data collection}

With the advance permission of a health center located in Osaka Prefecture, researchers verbally requested participation following 18-month health checkups. Mothers who gave consent received an anonymous, self-reported questionnaire, which was collected via postal service.

\section{5) Contents of survey}

The survey contained 20 questions in total, prepared for this study with reference to prior research. Subjects were asked to respond with respect to their infant's first year of life. Pretesting was conducted on four mothers with children aged 18 months.

\section{6) Method of analysis \& evaluation}

Each item of the self-reported responses was analyzed using the statistics software SPSS version 27.0. Additionally, inferential statistics were used to compare the differences between primipara/multipara and the presence/lack of instruction pertaining to experienced dangers. Inferential statistics were verified using the $\chi^{2}$ test or Fisher's exact test, with a significance level of less than 5\%.

7) Ethical considerations 
This study was conducted with the approval of the Osaka Medical and Pharmaceutical University Ethics Committee (Approval code: Nursing-142 2862, approved January 28,2020$)$. With the advance permission of a health center located in Osaka Prefecture, questionnaires and explanatory materials were distributed to mothers following their infants' 18-month health checkups. Consent was given in writing upon submission of the questionnaire. Subjects were informed of the study's title, the names of the involved research organizations, the name of the principal investigator, the purpose of the study, the method and term of the study, the reason they were selected to participate, that the benefit of their participation would be their contribution to the field of nursing and that there would be no direct profit or compensation for their cooperation, that personal information would be anonymized and coded in such a way that such information would not be personally identifiable, that anonymity would be maintained in any published data, the method of storage and disposal of personal information, the circumstances involving conflicts of interest with the research, and the availability of the researchers or related parties for consultation. The content of the questionnaire was carefully selected in order to minimize the burden on participants.

\section{Results}

\section{1) Questionnaire collection rate}

Questionnaires were given to 679 individuals, and 304 responses were received (collection rate of $44.8 \%$ ). Responses that contained partially incomplete or inappropriate answers were classified as no response during the analysis.

2) Subject characteristics (Tables 1-3)

Table 1 shows the number and age of children in each household, Table 2 shows the parents' employment status, and Table 3 shows the parents' bathing habits.

Table 1. Number of children and age of older children in household.

\begin{tabular}{cccc}
\hline & & $\#$ & $\%$ \\
\hline $\begin{array}{c}\text { Number of Children } \\
(\mathrm{n}=304)\end{array}$ & Second child or later & 159 & 52.3 \\
& First child & 145 & 47.7 \\
\hline & 1 year & 9 & 5.7 \\
Age of Older Children \\
$(\mathrm{n}=159)$ & 3 years & 4 & 2.5 \\
& 4 years & 53 & 15.1 \\
& 5 years & 34 & 33.3 \\
& 6 years & 20 & 21.4 \\
$7+$ years & 44 & 12.6 \\
& No response & 3 & 27.7 \\
\hline
\end{tabular}


Table 2. Parents' employment status $(\mathrm{n}=304)$.

\begin{tabular}{cccccc}
\hline \multicolumn{6}{c}{ Number (\%) } \\
\hline & Employed & Unemployed & On childcare leave & Other & No response \\
\hline Mothers & $135(44.4)$ & $117(38.5)$ & $40(13.2)$ & $3(1.0)$ & $9(3.0)$ \\
Fathers & $293(96.4)$ & $2(0.7)$ & $0(0.0)$ & $0(0.0)$ & $9(3.0)$ \\
\hline
\end{tabular}

Table 3. Parents' bathing habits $(\mathrm{n}=304)$.

\begin{tabular}{ccccccc}
\hline \multicolumn{7}{c}{ Number (\%) } \\
\hline $\begin{array}{c}\text { Bath } \\
\text { (daily) }\end{array}$ & $\begin{array}{c}\text { Shower } \\
\text { only }\end{array}$ & $\begin{array}{c}\text { Shower usually, } \\
\text { with occasional bath }\end{array}$ & $\begin{array}{c}\text { Shower in summer, } \\
\text { bath in winter }\end{array}$ & Other & $\begin{array}{c}\text { No } \\
\text { response }\end{array}$ \\
\hline Mothers & $181(59.5)$ & $11(3.6)$ & $29(9.5)$ & $77(25.3)$ & $1(0.3)$ & $6(2.0)$ \\
Fathers & $154(50.7)$ & $24(7.9)$ & $43(14.1)$ & $70(23.0)$ & $3(1.0)$ & $11(3.6)$ \\
\hline
\end{tabular}

159 subjects (52.3\%) were on their second child or later, while 145 were on their first (47.7\%). Of the 159 subjects who responded that they had two or more children, the most common ages were as follows: 53 subjects $(33.3 \%)$ had a five-year-old, $44(27.7 \%)$ had a child of age 7 or older, and $34(21.4 \%)$ had a five-year-old. For both mother and fathers, the 30 - 34 age group was the most common, with 131 (43.1\%) and 115 (37.8\%) respectively. 135 mothers (43.1\%) and 293 fathers (96.4\%) were employed. 40 mothers (13.2\%) were on maternity leave. The majority of mothers $(200,65.8 \%)$ gave birth in hospitals. 256 subjects (84.2\%) lived in nuclear families. Regarding bathing habits, 181 mothers (59.5\%) and 154 fathers (50.4\%) usually took a bath every day.

\section{3) State of children's bathing}

a) Bathing routine (Table 4)

The breakdown of subjects' bathing routines for their children is shown in Table 4 .

232 mothers (76.3\%) and 67 fathers (22.0\%) were the primary bath giver. 204 fathers $(67.1 \%)$ and 89 mothers (29.3\%) assisted with bathing.

The most common cleaning agent used for bathing was foamy soap, with 261 subjects $(85.9 \%)$. The most common washing implement was the hands $(270$ subjects, $88.8 \%$ ), followed by gauze (135 subjects, $44.4 \%$ ). 248 subjects (84.9\%) responded that they rinsed their infant with the showerhead, and 129 (39.5\%) rinsed by pouring water. The most common response concerning bathing equipment was that none was used (150 subjects, 49.3\%). Pieces of bathing equipment used were: bath seats (88 subjects, $28.9 \%$ ), bath mats (82 subjects, $27.0 \%)$, and neck-worn flotation rings (18 subjects, 5.9\%). 22 subjects (7.2\%) used other equipment such as baby bathtubs.

b) Dangers experienced (Table 5, Figure 1 and Figure 2)

Table 5 shows whether subjects experienced incidents or not, Figure 1 shows what dangers were experienced, and Figure 2 shows the situations in which incidents occurred.

213 subjects $(71.1 \%)$ responded that they had experienced incidents. 
Table 4. State of children's bathing $(\mathrm{n}=304)$.

\begin{tabular}{|c|c|c|c|}
\hline & & $\#$ & $\%$ \\
\hline \multirow{5}{*}{ Primary bath giver } & Mother & 232 & 76.3 \\
\hline & Father & 67 & 22.0 \\
\hline & Paternal grandfather & 1 & 0.3 \\
\hline & Siblings & 2 & 0.7 \\
\hline & Other & 2 & 0.7 \\
\hline \multirow{10}{*}{$\begin{array}{l}\text { Assists with bathing } \\
\text { (multiple response) }\end{array}$} & Mother & 89 & 29.3 \\
\hline & Father & 204 & 67.1 \\
\hline & Maternal grandmother & 45 & 14.8 \\
\hline & Maternal grandfather & 13 & 4.3 \\
\hline & Paternal grandmother & 11 & 3.6 \\
\hline & Paternal grandfather & 2 & 0.7 \\
\hline & Siblings & 35 & 11.5 \\
\hline & None & 28 & 9.2 \\
\hline & Other & 2 & 0.7 \\
\hline & No response & 1 & 0.3 \\
\hline \multirow{6}{*}{$\begin{array}{c}\text { Cleansers used } \\
\text { (multiple response) }\end{array}$} & None & 3 & 1.0 \\
\hline & Solid soap & 47 & 15.5 \\
\hline & Liquid soap & 33 & 10.9 \\
\hline & Foam soap & 261 & 85.9 \\
\hline & Body wash & 19 & 6.3 \\
\hline & Other & 2 & 0.7 \\
\hline \multirow{4}{*}{$\begin{array}{l}\text { Washing instruments } \\
\text { (multiple response) }\end{array}$} & Hands & 270 & 88.8 \\
\hline & Gauze & 135 & 44.4 \\
\hline & Towel & 18 & 5.9 \\
\hline & Other & 14 & 4.6 \\
\hline \multirow{3}{*}{$\begin{array}{c}\text { Rinsing method } \\
\text { (multiple response) }\end{array}$} & Shower & 258 & 84.9 \\
\hline & Pouring water & 120 & 39.5 \\
\hline & No response & 1 & 0.3 \\
\hline \multirow{6}{*}{$\begin{array}{l}\text { Bathing equipment } \\
\text { (multiple response) }\end{array}$} & None & 150 & 49.3 \\
\hline & Bath chair & 88 & 28.9 \\
\hline & Bath mat & 82 & 27.0 \\
\hline & Neck-worn flotation ring & 18 & 5.9 \\
\hline & Other & 22 & 7.2 \\
\hline & No response & 1 & 0.3 \\
\hline
\end{tabular}

Table 5. Experienced dangerous incidents $(n=304)$.

\begin{tabular}{ccc}
\hline & Number & $\%$ \\
\hline Yes & 216 & 71.1 \\
No & 88 & 28.9 \\
\hline
\end{tabular}




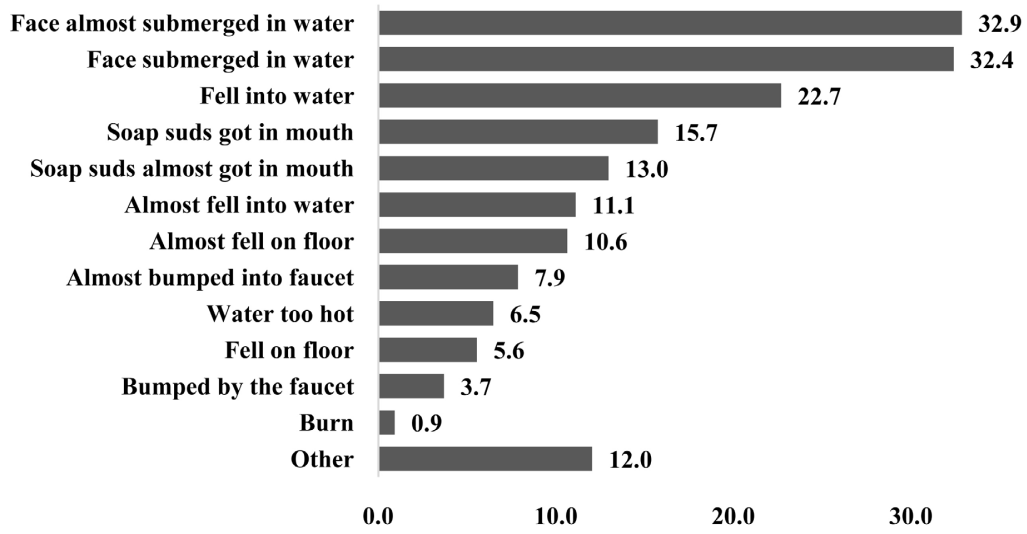

Figure 1. Incidents experienced $(\mathrm{n}=216)$ (Multipleresponse).

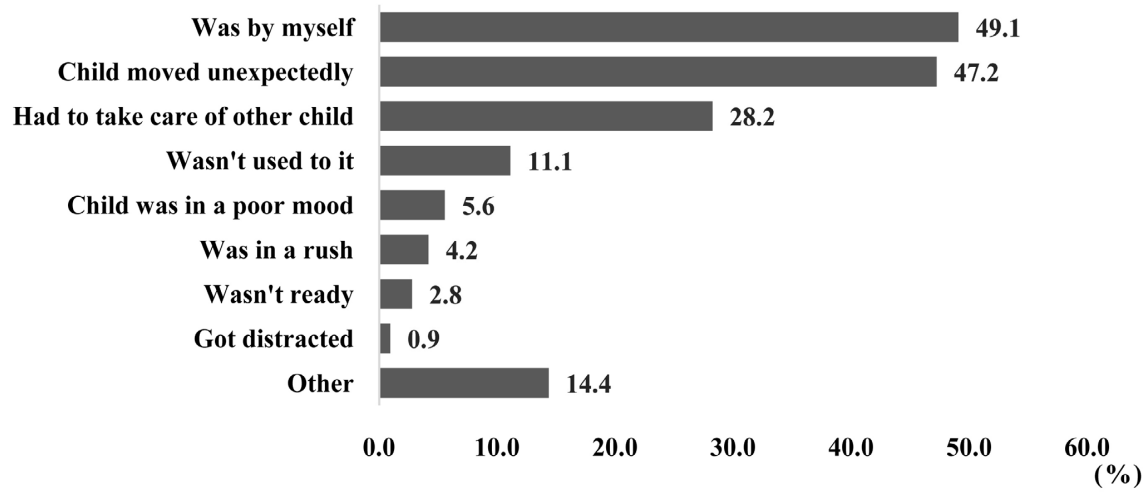

Figure 2. Situation during incidents $(n=216)$ (Multipleresponse).

Among mothers and their families who had experienced incidents, the most common were, in order of frequency, near-submersion of the face $(n=71$, $32.9 \%)$, submersion of the face $(\mathrm{n}=70,32.4 \%)$, and falling into bathwater $(\mathrm{n}=$ $49,22.7 \%)$. Similarly, the most common situations when incidents had occurred were giving the bath alone $(\mathrm{n}=106,49.1 \%)$, the child moving unexpectedly ( $\mathrm{n}=$ $102,47.2 \%)$, and taking care of other children $(\mathrm{n}=61,28.2 \%)$.

c) Measures taken to prevent incidents (Figure 3)

Figure 3 shows what measures were taken to prevent incidents.

Regarding methods of preventing incidents, the most common responses were: giving a bath when many people are around to help $(\mathrm{n}=109,35.9 \%)$, nothing ( $\mathrm{n}=97,31.9 \%)$, and using helpful bathing equipment such as bath seats $(\mathrm{n}=72,23.7 \%)$.

d) State of instruction (Table 6)

215 mothers and families (70.7\%) had not received instruction regarding the possible dangers that can occur during bathtime or the prevention of such dangers.

Table 6 shows the state of instruction regarding the dangers that can occur during bathtime and the prevention of such dangers. 


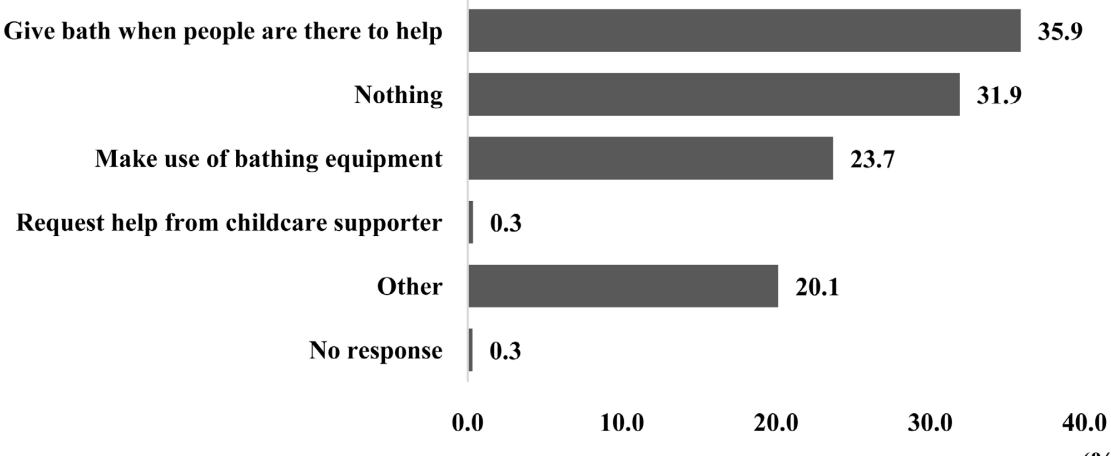

Figure 3. Measures taken against incidents $(\mathrm{n}=304)$ (Multipleresponse).

Table 6. State of instruction concerning possible bathing incidents.

\begin{tabular}{|c|c|c|c|}
\hline & & \# & $\%$ \\
\hline \multirow{3}{*}{$\begin{array}{c}\text { Content } \\
(\mathrm{n}=89) \\
\text { (multiple response) }\end{array}$} & What kinds of incidents can happen & 81 & 91.0 \\
\hline & Prevention methods & 43 & 48.3 \\
\hline & Other & 4 & 4.5 \\
\hline \multirow{6}{*}{$\begin{array}{l}\text { Place of instruction } \\
\qquad(\mathrm{n}=89) \\
\text { (multiple response) }\end{array}$} & Maternity classes & 35 & 39.3 \\
\hline & Parenting classes & 25 & 28.1 \\
\hline & During hospital stay after childbirth & 68 & 76.4 \\
\hline & Health exam & 12 & 13.5 \\
\hline & Home visit & 3 & 3.4 \\
\hline & Other & 5 & 5.6 \\
\hline \multirow{6}{*}{$\begin{array}{l}\text { Source of information } \\
\qquad(\mathrm{n}=304) \\
\text { (multiple response) }\end{array}$} & Internet & 114 & 37.5 \\
\hline & Maternity magazine/parenting materials & 104 & 34.2 \\
\hline & Nurse, midwife, or health professional & 122 & 40.1 \\
\hline & No information received & 55 & 18.1 \\
\hline & Other & 26 & 8.6 \\
\hline & No response & 12 & 3.9 \\
\hline
\end{tabular}

Among 89 respondents who received instruction, the content of the instruction included what kinds of incidents can happen $(n=81,91.0 \%)$ and prevention methods $(\mathrm{n}=43,48.3 \%$ ). The most common settings in which instruction was received were during the hospital stay after childbirth $(n=68,76.4 \%)$ and in maternity classes $(n=35,39.3 \%)$.

The most common sources of information concerning bathtime dangers were from a nurse/midwife/public health nurse $(\mathrm{n}=122,40.1 \%)$ and the Internet $(\mathrm{n}=$ $114,37.5 \%)$.

e) Comparison of incidents and situations between primipara/multipara and based on the presence of instruction (Table 7 and Table 8)

Table 7 compares the dangers experienced by primipara and multipara and the situations in which they were experienced. Table 8 compares the dangers 
Table 7. Comparison of experienced incidents and situations between Primipara and Multipara.

\begin{tabular}{|c|c|c|c|c|c|c|c|c|}
\hline & & \multirow{3}{*}{$\begin{array}{l}\text { Experienced } \\
\text { Incident }\end{array}$} & \multicolumn{4}{|c|}{ Number of Children } & \multirow{3}{*}{$x^{2}$} & \multirow{3}{*}{$\begin{array}{l}\text { Significant } \\
\text { difference }\end{array}$} \\
\hline & & & \multicolumn{2}{|c|}{$2^{\text {nd }}$ child +} & \multicolumn{2}{|c|}{$1^{\text {st }}$ child } & & \\
\hline & & & $\#$ & $\%$ & $\#$ & $\%$ & & \\
\hline \multirow{28}{*}{ Content } & Experienced & No & 48 & 30.2 & 40 & 27.6 & \multirow{2}{*}{0.250} & \multirow{2}{*}{ n.s. } \\
\hline & dangerous incident & Yes & 111 & 69.8 & 105 & 72.4 & & \\
\hline & \multirow{2}{*}{$\begin{array}{l}\text { Face almost } \\
\text { submerged }\end{array}$} & No & 121 & 76.1 & 112 & 77.2 & \multirow{2}{*}{0.055} & \multirow{2}{*}{ n.s. } \\
\hline & & Yes & 38 & 23.9 & 33 & 22.8 & & \\
\hline & \multirow{2}{*}{$\begin{array}{l}\text { Almost fell } \\
\text { into water }\end{array}$} & No & 149 & 93.7 & 131 & 90.3 & \multirow{2}{*}{1.182} & \multirow{2}{*}{ n.s. } \\
\hline & & Yes & 10 & 6.3 & 14 & 9.7 & & \\
\hline & \multirow{2}{*}{$\begin{array}{l}\text { Soap suds nearly } \\
\text { got in eyes }\end{array}$} & No & 141 & 88.7 & 135 & 93.1 & \multirow{2}{*}{1.775} & \multirow{2}{*}{ n.s. } \\
\hline & & Yes & 18 & 11.3 & 10 & 6.9 & & \\
\hline & \multirow{2}{*}{ Water too hot } & No & 154 & 96.9 & 136 & 93.8 & \multirow[b]{2}{*}{1.619} & \multirow[b]{2}{*}{ n.s. } \\
\hline & & Yes & 5 & 3.1 & 9 & 6.2 & & \\
\hline & \multirow{2}{*}{ Almost fell on floor } & No & 149 & 93.7 & 132 & 91.0 & \multirow{2}{*}{0.777} & \multirow{2}{*}{ n.s. } \\
\hline & & Yes & 10 & 6.3 & 13 & 9.0 & & \\
\hline & \multirow{2}{*}{$\begin{array}{l}\text { Almost bumped } \\
\text { into faucet }\end{array}$} & No & 152 & 95.6 & 135 & 93.1 & \multirow{2}{*}{0.894} & $n s$ \\
\hline & & Yes & 7 & 4.4 & 10 & 6.9 & & 11.0. \\
\hline & & No & 123 & 77.4 & 111 & 76.6 & & \\
\hline & 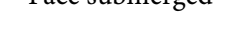 & Yes & 36 & 22.6 & 34 & 23.4 & 0.028 & n.s. \\
\hline & & No & 129 & 81.1 & 126 & 86.9 & & \\
\hline & Fell in water & Yes & 30 & 18.9 & 19 & 13.1 & 1.864 & n.s. \\
\hline & & No & 145 & 91.2 & 125 & 86.2 & & \\
\hline & Soap suds in eyes & Yes & 14 & 8.8 & 20 & 13.8 & 1.900 & n.s. \\
\hline & & No & 158 & 99.4 & 144 & 99.3 & & \\
\hline & DuाII & Yes & 1 & 0.6 & 1 & 0.7 & 0.004 & n.s. \\
\hline & & No & 155 & 97.5 & 137 & 94.5 & & \\
\hline & Fell on floor & Yes & 4 & 2.5 & 8 & 5.5 & 1.802 & n.s. \\
\hline & Bumped & No & 155 & 97.5 & 141 & 97.2 & & \\
\hline & into faucet & Yes & 4 & 2.5 & 4 & 2.8 & 0.017 & 11.0. \\
\hline & & No & 147 & 92.5 & 131 & 90.3 & & \\
\hline & Uther & Yes & 12 & 7.5 & 14 & 9.7 & 0.431 & n.s. \\
\hline & Was giving & No & 116 & 73.0 & 82 & 56.6 & 8986 & $* *$ \\
\hline & bath alone & Yes & 43 & 27.0 & 63 & 43.4 & 8.980 & \\
\hline & Whsin ruch & No & 152 & 95.6 & 143 & 98.6 & & \\
\hline Situation & 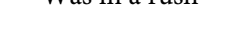 & Yes & 7 & 4.4 & 2 & 1.4 & 2.110 & 110.0. \\
\hline & Was taking care & No & 99 & 62.3 & 144 & 99.3 & 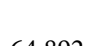 & \\
\hline & of other children & Yes & 60 & 37.7 & 1 & 0.7 & 04.892 & \\
\hline
\end{tabular}




\section{Continued}

\begin{tabular}{ccccccccc}
\hline Was distracted & No & 157 & 98.7 & 145 & 100.0 & & \\
& Yes & 2 & 1.3 & 0 & 0.0 & & \\
& No & 156 & 98.1 & 124 & 85.5 & & \\
Wasn't used to it & Yes & 3 & 1.9 & 21 & 14.5 & & \\
& No & 156 & 98.1 & 142 & 97.9 & & \\
Wasn't prepared & Yes & 3 & 1.9 & 3 & 2.1 & 0.013 & n.s. \\
& No & 151 & 95.0 & 141 & 97.2 & & \\
Child was in & Yes & 8 & 5.0 & 4 & 2.8 & & n.s. \\
poor mood & No & 113 & 71.1 & 89 & 61.4 & & \\
Child moved & Yes & 46 & 28.9 & 56 & 38.6 & & n.s. \\
unexpectedly & No & 141 & 88.7 & 132 & 91.0 & & \\
Other & Yes & 18 & 11.3 & 13 & 9.0 & & n.s. \\
& & & & & & & \\
\hline
\end{tabular}

${ }^{* *}$ : $\mathrm{p}<0.01$; n.s.: not significant.

Table 8. Comparison of incidents and situations based on presence of instruction.

\begin{tabular}{|c|c|c|c|c|c|c|c|c|}
\hline & & \multirow{3}{*}{$\begin{array}{l}\text { Experienced } \\
\text { Incident }\end{array}$} & \multicolumn{4}{|c|}{ Instruction given? } & \multirow{3}{*}{$x^{2}$} & \multirow{3}{*}{$\begin{array}{l}\text { Significan } \\
\text { difference }\end{array}$} \\
\hline & & & \multicolumn{2}{|c|}{ Yes } & \multicolumn{2}{|c|}{ No } & & \\
\hline & & & $\#$ & $\%$ & $\#$ & $\%$ & & \\
\hline \multirow{20}{*}{ Content } & Experienced & No & 23 & 25.8 & 65 & 30.2 & \multirow{2}{*}{0.590} & \multirow{2}{*}{ n.s. } \\
\hline & dangerous incident & Yes & 66 & 74.2 & 150 & 69.8 & & \\
\hline & \multirow{2}{*}{$\begin{array}{l}\text { Face almost } \\
\text { submerged }\end{array}$} & No & 69 & 77.5 & 164 & 76.3 & \multirow{2}{*}{0.055} & \multirow{2}{*}{ n.s. } \\
\hline & & Yes & 20 & 22.5 & 51 & 23.7 & & \\
\hline & \multirow{2}{*}{$\begin{array}{l}\text { Almost fell } \\
\text { into water }\end{array}$} & No & 81 & 91.0 & 199 & 92.6 & \multirow{2}{*}{0.207} & \multirow{2}{*}{ n.s. } \\
\hline & & Yes & 8 & 9.0 & 16 & 7.4 & & \\
\hline & \multirow{2}{*}{$\begin{array}{l}\text { Soap suds nearly } \\
\text { got in eyes }\end{array}$} & No & 81 & 91.0 & 195 & 90.7 & \multirow{2}{*}{0.007} & \multirow{2}{*}{ n.s. } \\
\hline & & Yes & 8 & 9.0 & 20 & 9.3 & & \\
\hline & \multirow{2}{*}{ Water too hot } & No & 84 & 94.4 & 206 & 95.8 & \multirow{2}{*}{0.294} & \multirow{2}{*}{ n.s. } \\
\hline & & Yes & 5 & 5.6 & 9 & 4.2 & & \\
\hline & \multirow{2}{*}{ Almost fell on floor } & No & 79 & 88.8 & 202 & 94.0 & \multirow{2}{*}{2.424} & \multirow{2}{*}{ n.s. } \\
\hline & & Yes & 10 & 11.2 & 13 & 6.0 & & \\
\hline & \multirow{2}{*}{$\begin{array}{l}\text { Almost bumped } \\
\text { into faucet }\end{array}$} & No & 81 & 91.0 & 206 & 95.8 & \multirow{2}{*}{2.750} & \multirow{2}{*}{ n.s. } \\
\hline & & Yes & 8 & 9.0 & 9 & 4.2 & & \\
\hline & \multirow{2}{*}{ Face submerged } & No & 68 & 76.4 & 166 & 77.2 & \multirow{2}{*}{0.023} & \multirow{2}{*}{ n.s. } \\
\hline & & Yes & 21 & 23.6 & 49 & 22.8 & & \\
\hline & \multirow{2}{*}{ Fell in water } & No & 73 & 82.0 & 182 & 84.7 & \multirow{2}{*}{0.322} & \multirow{2}{*}{ n.s. } \\
\hline & & Yes & 16 & 18.0 & 33 & 15.3 & & \\
\hline & Soap suds & No & 78 & 87.6 & 192 & 89.3 & & \\
\hline & in eyes & Yes & 11 & 12.4 & 23 & 10.7 & 0.07 & . \\
\hline
\end{tabular}




\section{Continued}

\begin{tabular}{|c|c|c|c|c|c|c|c|c|}
\hline & \multirow{2}{*}{ Burn } & No & 89 & 100.0 & 213 & 99.1 & \multirow{2}{*}{0.833} & \multirow{2}{*}{ n.s. } \\
\hline & & Yes & 0 & 0.0 & 2 & 0.9 & & \\
\hline & \multirow{2}{*}{ Fell on floor } & No & 85 & 95.5 & 207 & 96.3 & \multirow{2}{*}{0.099} & \multirow{2}{*}{ n.s } \\
\hline & & Yes & 4 & 4.5 & 8 & 3.7 & & \\
\hline & \multirow{2}{*}{$\begin{array}{l}\text { Bumped } \\
\text { into faucet }\end{array}$} & No & 88 & 98.9 & 208 & 96.7 & \multirow{2}{*}{1.117} & \multirow{2}{*}{ n.s } \\
\hline & & Yes & 1 & 1.1 & 7 & 3.3 & & \\
\hline & \multirow{2}{*}{ Other } & No & 82 & 92.1 & 196 & 91.2 & \multirow{2}{*}{0.076} & \multirow{2}{*}{ n.s } \\
\hline & & Yes & 7 & 7.9 & 19 & 8.8 & & \\
\hline & \multirow{2}{*}{$\begin{array}{l}\text { Was giving } \\
\text { bath alone }\end{array}$} & No & 56 & 62.9 & 142 & 66.0 & \multirow{2}{*}{0.271} & \multirow{2}{*}{ n.s } \\
\hline & & Yes & 33 & 37.1 & 73 & 34.0 & & \\
\hline & \multirow{2}{*}{ Was in a rush } & No & 85 & 95.5 & 210 & 97.7 & \multirow{2}{*}{1.031} & \multirow{2}{*}{ n.s } \\
\hline & & Yes & 4 & 4.5 & 5 & 2.3 & & \\
\hline & \multirow{2}{*}{$\begin{array}{l}\text { Was taking care } \\
\text { of other children }\end{array}$} & No & 64 & 71.9 & 179 & 83.3 & \multirow{2}{*}{5.052} & \multirow{2}{*}{ * } \\
\hline & & Yes & 25 & 28.1 & 36 & 16.7 & & \\
\hline & \multirow{2}{*}{ Was distracted } & No & 89 & 100.0 & 213 & 99.1 & \multirow{2}{*}{0.833} & \multirow{2}{*}{ n.s. } \\
\hline & & Yes & 0 & 0.0 & 2 & 0.9 & & \\
\hline \multirow{10}{*}{ Situation } & \multirow{2}{*}{ Wasn't used to it } & No & 77 & 86.5 & 203 & 94.4 & \multirow{2}{*}{5.405} & \multirow{2}{*}{ * } \\
\hline & & Yes & 12 & 13.5 & 12 & 5.6 & & \\
\hline & \multirow{2}{*}{ Wasn't prepared } & No & 88 & 98.9 & 210 & 97.7 & \multirow{2}{*}{0.470} & \multirow{2}{*}{ n.s. } \\
\hline & & Yes & 1 & 1.1 & 5 & 2.3 & & \\
\hline & \multirow{2}{*}{$\begin{array}{c}\text { Child was in } \\
\text { poor mood }\end{array}$} & No & 87 & 97.8 & 205 & 95.3 & $50=0$ & \\
\hline & & Yes & 2 & 2.2 & 10 & 4.7 & 0.703 & 110.3. \\
\hline & Child moved & No & 55 & 61.8 & 147 & 68.4 & דרת & \\
\hline & unexpectedly & Yes & 34 & 38.2 & 68 & 31.6 & & 11.0. \\
\hline & & No & 83 & 93.3 & 190 & 88.4 & & \\
\hline & Gines & Yes & 6 & 6.7 & 25 & 11.6 & 1.041 & In.s. \\
\hline
\end{tabular}

*: $\mathrm{p}<0.05$; n.s.: not significant.

experienced based on the presence or absence of instruction concerning those dangers and the situations in which they were experienced.

When comparing primipara and multipara, more mothers and families with one child responded they gave a bath alone or were not used to giving baths, while significantly more mothers and families with multiple children responded that they were taking care of another child. Furthermore, when comparing by the presence of instruction, significantly higher percentages of subjects who had received instruction responded that they were looking after other children or not used to giving baths when an incident occurred than those who had not.

f) Comparison of prevention measures between primipara/multipara and based on presence of instruction (Table 9 and Table 10) 
Table 9. Comparison of prevention measures between Primipara and Multipara.

\begin{tabular}{|c|c|c|c|c|c|c|c|}
\hline & \multirow{3}{*}{$\begin{array}{l}\text { Experienced } \\
\text { Incident }\end{array}$} & \multicolumn{4}{|c|}{ Number of Children } & \multirow{3}{*}{$x^{2}$} & \multirow{3}{*}{$\begin{array}{l}\text { Significant } \\
\text { difference }\end{array}$} \\
\hline & & \multicolumn{2}{|c|}{$2^{\text {nd }}$ child +} & \multicolumn{2}{|c|}{$1^{\text {st }}$ child } & & \\
\hline & & $\#$ & $\%$ & $\#$ & $\%$ & & \\
\hline \multirow{2}{*}{ No measures taken } & No & 108 & 67.9 & 99 & 68.3 & \multirow{2}{*}{0.004} & \multirow{2}{*}{ n.s. } \\
\hline & Yes & 51 & 32.1 & 46 & 31.7 & & \\
\hline \multirow{2}{*}{$\begin{array}{l}\text { Give bath when people } \\
\text { are there to help }\end{array}$} & No & 104 & 65.4 & 91 & 62.8 & \multirow{2}{*}{0.232} & \multirow{2}{*}{ n.s. } \\
\hline & Yes & 55 & 34.6 & 54 & 37.2 & & \\
\hline \multirow{2}{*}{$\begin{array}{c}\text { Make use of } \\
\text { bathing equipment }\end{array}$} & No & 124 & 78.0 & 108 & 74.5 & \multirow{2}{*}{0.515} & \multirow{2}{*}{ n.s. } \\
\hline & Yes & 35 & 22.0 & 37 & 25.5 & & \\
\hline \multirow{2}{*}{$\begin{array}{l}\text { Request help from } \\
\text { childcare supporter }\end{array}$} & No & 158 & 99.4 & 145 & 100.0 & \multirow{2}{*}{0.915} & \multirow{2}{*}{ n.s. } \\
\hline & Yes & 1 & 0.6 & 0 & 0.0 & & \\
\hline \multirow{2}{*}{ Other } & No & 122 & 76.7 & 121 & 83.4 & \multirow{2}{*}{2.134} & \multirow{2}{*}{ n.s. } \\
\hline & Yes & 37 & 23.3 & 24 & 16.6 & & \\
\hline
\end{tabular}

n.s.: not significant.

Table 10. Comparison of prevention measures based on presence of instruction.

\begin{tabular}{|c|c|c|c|c|c|c|c|}
\hline & \multirow{3}{*}{$\begin{array}{l}\text { Experienced } \\
\text { Incident }\end{array}$} & \multicolumn{4}{|c|}{ Instruction given? } & \multirow{3}{*}{$x^{2}$} & \multirow{3}{*}{$\begin{array}{l}\text { Significant } \\
\text { difference }\end{array}$} \\
\hline & & \multicolumn{2}{|c|}{ Yes } & \multicolumn{2}{|c|}{ No } & & \\
\hline & & $\#$ & $\%$ & \# & $\%$ & & \\
\hline \multirow[b]{2}{*}{ No measures taken } & No & 68 & 76.4 & 139 & 64.7 & \multirow[b]{2}{*}{4.002} & \multirow[b]{2}{*}{ * } \\
\hline & & 21 & 23.6 & 76 & 35.3 & & \\
\hline \multirow{2}{*}{$\begin{array}{l}\text { Give bath when people } \\
\text { are there to help }\end{array}$} & No & 48 & 53.9 & 147 & 68.4 & \multirow{2}{*}{5.706} & \multirow{2}{*}{ * } \\
\hline & Yes & 41 & 46.1 & 68 & 31.6 & & \\
\hline \multirow{2}{*}{$\begin{array}{c}\text { Make use of } \\
\text { bathing equipment }\end{array}$} & No & 63 & 70.8 & 169 & 78.6 & \multirow{2}{*}{2.129} & \multirow{2}{*}{ n.s. } \\
\hline & Yes & 26 & 29.2 & 46 & 21.4 & & \\
\hline \multirow{2}{*}{$\begin{array}{l}\text { Request help from } \\
\text { childcare supporter }\end{array}$} & No & 88 & 98.9 & 215 & 100.0 & \multirow{2}{*}{2.424} & \multirow{2}{*}{ n.s. } \\
\hline & Yes & 1 & 1.1 & 0 & 0.0 & & \\
\hline \multirow{2}{*}{ Other } & No & 75 & 84.3 & 168 & 78.1 & \multirow{2}{*}{1.475} & \multirow{2}{*}{ n.s. } \\
\hline & Yes & 14 & 15.7 & 47 & 21.9 & & \\
\hline
\end{tabular}

${ }^{*}$ : $\mathrm{p}<0.05$; n.s.: not significant.

Table 9 compares the primipara and multipara concerning measures for preventing incidents. Table 10 compares subjects based on whether they had received instruction on these dangers.

No significant differences were found when comparing the measures taken between primipara and multipara. When comparing groups based on the presence of instruction, significantly more subjects who had received instruction gave a bath when there was someone to help than those who did not. Significantly more subject who had not received instruction responded that they took 
no preventative measures than those who had.

\section{4) Opinions and requests for nurses concerning the dangers of infant} bathing

Opinions and requests for nursing professionals included proper infant bathing technique, concrete examples of dangers, preventative measures, methods and caution points for bathing multiple children at once, methods and caution points for giving a bath alone, methods and caution points of bathing together with the baby, proper use and caution points of bathing products, and the timing/frequency of instruction.

Among mothers with multiple children and their families, opinions surfaced in categories such as methods and caution points for bathing multiple children at the same time, methods and caution points for giving a bath alone, and concrete examples of dangers.

Forming the category of methods and caution points for bathing multiple children at the same time were responses such as: "My oldest is still small and can't bathe by himself, so when I put them in together my hands are full enough with just him.", "Bathing with my kids is very difficult. Even one child is plenty, so it would have been a great help if I had gotten some advice for bathing more than one at a time.", and "I can handle looking after my first by [him] self, but two is too much. I wish I had been taught a good way to do it or what order is best-something I could put into practice right away."

Forming the category of methods and caution points for giving a bath alone were responses such as: "When I give my baby a bath by myself $1 \mathrm{~m}$ so nervous even from just washing my hair, I can't stand it. I can't even close my eyes.", "I would have liked if someone explained step-by-step how to do it by myself.", and "I wish I knew what to be careful of when I have to give my baby a bath by myself once they start moving around and can stand while holding on to something, and what to do with the baby while P $m$ washing myself."

Forming the category of concrete examples of dangers were responses such as: "I wasn't told about any dangers (that I remember). I think a kind of video course with examples of what can happen would be good to have.", "Hearing about everyone's close calls would be a good learning experience.", and "I had never heard about bathtime dangers, so day-to-day I never really thought about it. Whenever something dangerous happened, it always worked out, so I count myself lucky, but it even just being told about past incidents would be great for being prepared." Subjects also expressed interest in concrete methods and caution points for using bathing goods.

Among mothers with one child and their families, opinions surfaced in categories such as concrete examples of dangers, bathing methods, and methods and caution points for giving a bath alone.

Forming the category of concrete examples of dangers were responses such as: "I don't remember ever being asked at checkups about our bathtime routine. $1 \mathrm{~m}$ careful, but $P d$ like the kind of incidents that have happened to be common knowledge. There are unique dangers for different ages, so it doesn't apply only 
to babies. I would like there to be well-put-together materials to help with that.", "Even if you think you' re being careful and you think you' re watching, in an actual situation there's a lot you can miss. I wish they would give us examples to learn from.", and "My baby almost drowned even though the water wasn't deep. I wish someone would have told me about what can happen."

Forming the category of bathing methods are responses such as: "When I was in the hospital they told me how to give the baby a bath and I practiced, but when I bathed together with by baby I was nervous because I didn't know how to best do it safely. I wish they had taught me that.", "They taught me how to use a baby bathtub, and it's easy to find things online, but I didn't know what to do next.", and "I didn't have any opportunity to learn what to do after my baby grows out of the baby bath [and we start to use the regular tub/ shower.]"

Forming the category of methods and caution points for giving a bath alone were responses such as: "Many mothers have to put in the majority of the effort of raising children, so I think most of them have had close calls when bathing their babies by themselves. Of course, things will always happen, but I think we'll be more aware of the risks of death and injury if health professionals emphasize that education.", "I wish they had taught me what to do after coming home with the baby and how to be more efficient at bathtime. When you have to do it all yourself, being slow and clumsy leads to more risk of something happening.", and " I wished someone told me the best order when I take a bath with my baby. Washing myself first and then my baby after $1 \mathrm{~m}$ finished, for example."

\section{Observations}

\section{1) Subject characteristics and background}

According to the 2019 Comprehensive Survey of Living Conditions [8], 82.5\% of households were nuclear households. The percentage of nuclear households in this study was similar, at $84.2 \%$.

Additionally, the same survey [8] reported that the employment status in 2019 for mothers whose youngest child was one year old was full-time for $33.6 \%$, part-time/temporary for $19.3 \%$ and unemployed for $41.6 \%$. This study showed a situation similar to that shown by the national survey, with $57.6 \%$ of mothers being employed, including those on childcare leave.

Over half of mothers and fathers said they bathe in the tub every day. Bathing is an essential everyday custom in Japan; bathing customs affect perceptions about bathing and cultural factors such as lifestyle. Bathing styles change based on country and region, and also generation. In present-day Japan, the custom of bathing with only a shower has become more prevalent. However, compared to other countries, the custom of bathing in a tub nearly every day may be thought of as characteristic of Japan. Because of this, it is very common to use a tub of hot water when bathing infants as well, which may present many opportunities for incidents to occur.

2) The state of bathing 
In Japan, the amount of time women spend on childcare and housework is longer than in Western countries. These long hours create a burden for women. In this study, $76.3 \%$ of mothers and $22.0 \%$ of fathers identified as a primary bath giver, while $67.1 \%$ of fathers and $29.3 \%$ of mothers identified as having an assisting role in bathing. Bathing can be considered an aspect of childcare that fathers have an easy time participating in. However, $96.4 \%$ of fathers work, and the current working conditions in Japan make it difficult for many fathers to participate in childcare considering the life rhythms of an infant. Furthermore, fathers have few opportunities to seek guidance from childcare specialists. There have been cases of near-drownings while babies were in the bath with their father, and a greater number of cases in which the baby was in the care of someone other than the mother have occurred than cases in which the mother was present [9]. A possible reason for this may be that childcare instruction is heavily focused on mothers; inadequate family-oriented instruction leads to other members of the family having inadequate knowledge and thus an increase in incidents. It can be surmised that excessive urging from specialists for mothers and families to participate in infant care places a burden on fathers. It is important to promote cooperation from the commercial sector so fathers can participate in infant care, to support mothers so they can comfortably take care of the child by themselves, and to create an environment in which fathers can find information and consult as needed in their limited time.

The most common washing solvent used was foaming soap (85.9\%). The most common washing implements were the hand (88.8\%) followed by gauze towels (44.4\%). The most common methods of rinsing were with the shower head (84.9\%) and by pouring water (39.5\%). Self-foaming soap may be the most common due to the importance of lathering the soap when washing, and because it may be difficult to do this while holding the baby. By placing a foaming soap dispenser in the proper location, one can hold the baby with one arm and access the soap without taking their eyes off of it, reducing the risk of an accident.

When rinsing, it is important not to leave any residue from the cleanser on the skin. Using the showerhead is an effective way to adequately rinse in modern homes where a showerhead has become commonplace. Water temperature can also be an issue, so it may be necessary to keep the shower equipment in good repair to ensure safety.

$49.3 \%$ of respondents reported not using bathing equipment, which was the largest group. Equipment used was bath seats (28.9\%), bath mats (27.0\%), neck-worn flotation rings (5.9\%), and baby tubs/other (7.2\%). In recent years, many baby care goods have come to the market, offering a variety of convenient products. Bath seats and mats may help when bathing with one's baby by providing a place for the baby while the mother or family member washes themself. Using these products may offer a sense of comfort and safety. However, when using these products, a short lapse of attention can lead to a major incident. Kato [10] reports that a top incident involving infants younger than 1 year of age was 
near-drowning involving bath equipment. There have been numerous other reports of accidents involving bath equipment, including those involving neck-worn flotation rings and bathe seats [7]. The public must be educated on the proper application of such equipment, on the risks to their use, and on the prevention of those risks.

\section{3) Incidents experienced}

$71.1 \%$ of mothers and families experienced dangers involved with bathing. The most common dangers experienced by these families were, in order from most to least frequent, the baby's face being nearly submerged (32.9\%), the baby's face being submerged (32.4\%), and the baby falling into the water (22.7\%).

According to previous research, the number of bathtime incidents involving infants below the age of one year has been reported at 143 times the number involving children age 5 - 19 years old [11], and five out of eight drowning cases were younger than one year old [12]. Furthermore, there have been many reports of non-fatal drownings such as while using a neck-worn flotation ring [13] [14] [15] [16], slipping into the tub and inhaling water while the mother was shampooing her hair, [15] and drowning in the tub while bathing with family [17]. The top reported incidents experienced by mothers and their families reported this study was also related to drowning; the danger of drowning in the tub can be considered a major risk factor.

The most common situations in which an incident occurred were when giving the bath alone (49.1\%), the baby moving unexpectedly (47.2\%), and looking after another child (28.2\%). Related to these incidents are the characteristic trait of children to injure themselves through being unaware of the danger of their surroundings due to physical and mental immaturity, the dangers changing along with their development and their unawareness of the accidents that can occur due to this trait, and their tendency to lose their balance and fall over, among other things. Furthermore, early infancy is a period of rapid development, with new behaviors emerging without warning, such as turning over, sitting up, crawling, grasping objects to stand up, etc. They are also prone to behave in ways adults would not expect. Because of this, it can be thought that the most commonly reported situations (giving bath alone, the baby moved unexpectedly, and looking after other children) are particularly high-risk.

$70.7 \%$ of mothers and families had not received guidance about possible bathtime dangers or their prevention. Furthermore, the large number of opinions of and demands for nursing professionals (such as bathing methods, examples of bathtime dangers, prevention measures, methods and caution points of bathing multiple children at once, measures and caution points of giving a bath alone, uses and caution points of bathing equipment, and instruction timing/frequency) suggest a need for guidance from these professionals. While some of the dangers that can occur during bathtime can be prevented via an adult exercising caution, families being unaware how common childhood accidents are due to a lack of guidance, the behavioral changes that come with development, and the unpredictability of possible accidents are likely contributing factors to the occurrence 
of accidents involving infants. Families have few opportunities to obtain valuable guidance. Even among these, there are fewer still to learn about bathtime dangers, so it is necessary to provide specific guidance on potential dangers and their prevention. The prevention measures in this study (such as bathing when there are others around to help and using helpful products such as bath seats) may be helpful to mothers and families in the future and including them in instruction for the prevention of bathtime incidents may be effective. Additionally, since the sources of information regarding bathtime dangers were nurses/midwives/public health nurses (40.1\%) and the Internet (37.5\%), there may be a demand for instructional tools that enable mothers and families to easily obtain accurate information during their daily lives.

When we compared which dangers were experienced by one-child and multiple-child mothers and the situations when they occurred, significantly more single-child mothers and their families answered that they gave their baby a bath by themselves and that they were not accustomed to bathing an infant, while significantly more multiple-child mothers and their families answered that they were taking care of other children. It can be inferred that inexperience is linked to accidents in single-child families in particular. Considering bathing methods were among the demands single-child mothers and families had for the nursing profession, it can be assumed that without instruction in the basics, families' lack of practice in proper bathing procedure leads to incidents. It can also be gathered that in families with multiple children there are numerous opportunities for incidents to occur when parents are distracted because multiple children are in the bath at the same time. Reports of accidents related to siblings bathing together [18] [19] or occurring while the mother was distracted with an older sibling [20] have appeared in previous research. That methods and caution points of bathing multiple children at once was a concern expressed to nurses by multiple-child families leaves little doubt that families who must keep an eye on both an infant and their older children and have little choice but to bathe them together feel a sense of anxiety over the danger of bathing multiple children at the same time.

Furthermore, when we compared by the presence of instruction, significantly higher percentages of subjects who had received instruction responded that they were looking after other children or not used to giving baths when an incident occurred than those who had not. This suggests that the current instruction given is inadequate for preventing incidents when multiple children are being taken care of or the parent is not yet used to giving baths. It is necessary to explain what kinds of dangers can occur in concrete terms and how to prevent them, while considering the situation of each individual household.

There were no significant differences when we compared the preventative measures taken by primipara and multipara. Furthermore, when we compared these measures by the presence of instruction, a significantly higher percentage of subjects who had received instruction waited to bathe their child until someone was there to help than families who did not. Likewise, a significantly higher 
percentage of subjects who had not received instruction took no measures than those who had. Thus, receiving instruction may lead to greater awareness and execution of preventative measures such as lending assistance.

\section{Future Matters}

This study has revealed the state of dangers encountered by Japanese mothers and families in the course of bathing their infants, the situations in which they occur, and preventative measures are taken. Looking to the future, these trends should be compared with those of other countries. Concrete measures that nurses can take should be considered based on the results of this research, and an intervention study conducted.

\section{Conclusions}

The objective of this study was to reveal as-of-yet unknown bathtime-related dangers experienced by mothers and families in Japan, spurred by the need for measures to help mothers and families bathe their infants safely and with confidence. As a result, we have arrived at the following observations:

1) $70 \%$ of mothers and families have experienced bathtime-related dangers or incidents.

2) Among mothers and families who have experienced bathtime-related dangers, the most common was the infant's face's being nearly submerged, the infant's face's being fully submerged, and the infant's falling into the water, in that order.

3) The most common situations in which bathtime incidents occurred were when giving a bath alone, when the child moved unexpectedly, and when looking after other children, in that order.

4) Approximately $70 \%$ of mothers and families have not received guidance on possible bathtime incidents or their prevention.

5) For single-child mothers, in particular, a lack of childcare skills and inexperience is linked to the occurrence of bathtime incidents, while for multiple-child mothers bathing multiple children at the same time causes distraction and leads to incidents.

6) Even with instruction, subjects experienced incidents while tending to multiple children or due to inexperience.

7) Mothers and families are experiencing bathtime incidents, and prevention is necessary. The use of tools to allow mothers and families to easily obtain accurate information in the course of instruction is desirable.

\section{Acknowledgements}

We would like to extend our sincere gratitude to the mothers and families who so graciously offered their valuable time to assist in our study, the many helpful staff of the health center, and all who lent us their guidance along the way.

This study was conducted with the assistance of the JSPS Scientific Research 
Grant (JP20K 19151).

Parts of this study were presented at the $23^{\text {rd }}$ Congress of the Japan Society of Maternity Nursing.

\section{Conflicts of Interest}

There are no conflicts of interest related to this study.

\section{References}

[1] Ministry of Health, Labour and Welfare (2015) Annual Health, Labour and Welfare Report 2015.

[2] Naito, N. (2000) Regional Childcare Assistance. The Japanese Journal of Perinatal Care, 19, 112-121.

[3] Nagasaka, N. (2002) The State of Anxiety about Childcare: Raising Children behind the Closed Doors of the Home. Human Mind, 103, 50-56.

[4] Ministry of Health, Labour and Welfare (2020) Survey of Medical Institutions. 21-23. https://www.mhlw.go.jp/toukei/saikin/hw/iryosd/19/dl/03byouin01.pdf

[5] Chikazawa, S. and Sasaki, A. (2019) A Literature Review on Newborn and Infant Bathing Accidents. Journal of the Japan Maternal and Infant Care Association, 12, 83-90.

[6] Ministry of Health, Labour and Welfare (2020) Vital Statistics. https://www.mhlw.go.jp/toukei/saikin/hw/jinkou/geppo/nengai19/dl/h7.pdf

[7] Chikazawa, S. and Sasaki, A. (2020) Study on Troubles of Primiparas and Multiparas in Bathing Neonates and Infants. Journal of the Japan Maternal and Infant Care Association, 13, 25-36.

[8] Ministry of Health, Labour and Welfare (2020) Comprehensive Survey of Living Conditions. 6.

https://www.mhlw.go.jp/toukei/saikin/hw/k-tyosa/k-tyosa19/dl/02.pdf

[9] Hama, K. and Watanabe, R. (2007) A Study on the Related Factors of the Occurring Incidence or Accidents within 1.5 Year-Old Children Who Had Medical Examinations. The Journal of Child Health, 66, 10-15.

[10] Kato, Y., Takamine, C., Nakatsuji, H., et al. (2013) An Accidental Injury in Infancy Happens by the Child Care Items. The Journal of Child Health, 72, 267-273.

[11] Wallis, B.A., Watt, K., Franklin, R.C., Nixon, J.W., et al. (2015) Where Children and Adolescents Drown in Queensland: A Population-Based Study. BMJ Open, 5, e008959. https://doi.org/10.1136/bmjopen-2015-008959

[12] Sibert, J., John, N., Jenkins, D., et al. (2005) Drowning of Babies in Bath Seats: Do They Provide False Reassurance? Child: Care, Health and Development, 31, 255-259. https://doi.org/10.1111/j.1365-2214.2005.00516.x

[13] Tashiro, H., Mizukami, T. and Harada, M. (2017) A Case of Hyperbaric Oxygen Therapy for a 10-Month-Old Infant Who Drowned in the Bathtub. The Medical Journal of National Hospital Organization Kumamoto Medical Center, 16, 147-152.

[14] Someya, M. and Tsuji, S. (2016) Drowning While Using Neck Float Ring. Journal of Japanese Society of Emergency Pediatrics, 15, 412-414.

[15] Kato, Y., Oya, N., Nakatsuji, H., et al. (2015) Report Derived from the Investigation Papers about the Accidental Injuries of Infants, Which Happened at 0 Year Old and 1 Year Old Children-Those Were Caused by Their Parents' Little Understanding of the Riskiness and Infants' Movements beyond Their Expectations. The Journal of 
Child Health, 74, 712-718.

[16] Akiyama, R., Hiramoto, R., Komori, I., et al. (2010) Retrospective Analysis of 21 Drowning Cases. Japanese Journal of Pediatrics, 63, 1189-1195.

[17] Furuya, S., Nagai, S., Kirino, T., et al. (2012) Two Infantile Cases Performed Hypothermia Therapy after Cardiac Arrest of Drowning. The Medical Journal of Kagawa National Children's Hospital, 1, 36-40.

[18] Somers, G.R., Chiasson, D.A. and Smith, C.R. (2006) Pediatric Drowning: A 20-Year Review of Autopsied Cases: III. Bathtub Drownings. The American Journal of Forensic Medicine and Pathology, 27, 113-116. https://doi.org/10.1097/01.paf.0000220981.54069.b8

[19] Thompson, K.M. (2003) The Role of Bath Seats in Unintentional Infant Bathtub Drowning Deaths. Medscape General Medicine, 5, 36.

[20] Byard, R., de Koning, C., Blackbourne, B., et al. (2001) Shared Bathing and Drowning in Infants and Young Children. Journal of Paediatrics and Child Health, 37, 542-544. https://doi.org/10.1046/j.1440-1754.2001.00704.x 